Rev. Adm. Saúde - Vol. 18, № 73, out. - dez. 2018

http://dx.doi.org/10.23973/ras.73.110

RELATO DE CASO

\title{
Diagnóstico situacional de uma unidade de emergência cardiológica para avaliação da qualidade do serviço e da assistência ao paciente
}

Situational diagnosis of a cardiological emergency unit to evaluate the quality of service and patient care

\section{Ana Cláudia Becker, Raphael Kaeriyama e Silva, Raphael Tadashi Kaneko}

1. Gerontóloga. Aprimoranda do Programa de Estudos Avançados em Administração Hospitalar e Sistemas de Saúde (PROAHSA) do Hospital das Clínicas da Faculdade de Medicina da USP (HCFMUSP), São Paulo SP

2. Médico. Médico residente do PROAHSA do HCFMUSP, São Paulo SP

3. Médico. Médico preceptor do PROAHSA do HCFMUSP, São Paulo SP

\section{RESUMO}

A unidade de emergência cardiológica é tida como um serviço de referência pela natureza serviço prestado. No entanto, na busca pela melhoria do atendimento, deve-se usar indicadores que possibilitem a avaliação da qualidade do serviço. Embora não seja o único, a satisfação do paciente tem sido usada como indicador de qualidade já que orienta a análise da assistência através da mensuração qualitativa da percepção do paciente sobre a sua estadia hospitalar.

O objetivo deste trabalho é realizar o diagnóstico situacional do setor de emergência de um hospital cardiológico, de forma a avaliar a qualidade da assistência fornecida ao paciente e do serviço prestado pelos profissionais, baseado na satisfação percebida da unidade. Para tanto, foram elaborados dois questionários baseados na Escala NPS (Net Promoter Scale), cujo 
objetivo é mensurar a satisfação e a fidelidade dos consumidores ou usuários de uma empresa ou serviço.

No caso dos profissionais obtivemos $40 \%$ das respostas para promotores, $38 \%$ para neutros e $22 \%$ para detratores. Assim, o índice final do NPS para os profissionais foi de $18 \%$, sendo possível classificá-lo na zona de aperfeiçoamento. Já para os pacientes, obtivemos $62 \%$ das respostas para promotores, 35\% para neutros e 3\% para detratores e o índice final do NPS foi de $58 \%$, sendo possível classificá-lo na zona de qualidade.

Ressalta-se a disparidade entre os dois clientes da unidade de emergência cardiológica, profissionais e pacientes, o que demonstra que os pacientes são mais propensos a indicarem o serviço para outros clientes quando comparados aos profissionais. Sugere-se então, uma melhor compreensão acerca dos motivos pelos quais os resultados dos questionários aplicados não tenham atingido a zona de excelência.

O diagnóstico situacional foi realizado através da análise observacional da equipe, dos resultados dos questionários aplicados e do estudo dos casos de ouvidoria. Os pontos fracos e oportunidades de melhoria encontrados estão ligados principalmente a recursos humanos reduzidos, grande demanda de usuários e estrutura física antiga da unidade.

Palavras-chave: gestão de qualidade em saúde; satisfação do paciente; pessoal de saúde; serviços de atendimento de emergência

\section{ABSTRACT}

The cardiological emergency unit is taken as a referral service by the nature of service provided. However, in the search for better service, indicators should be used to evaluate the quality of service. Although not the only one, patient satisfaction has been used as an indicator of quality since it guides the analysis of care through the qualitative measurement of the patient's perception about his or her hospital stay.

The objective of this study is to perform the situational diagnosis of the emergency department of a cardiology hospital, to evaluate the quality of the care provided to the patient and the service provided by the professionals, based on the perceived satisfaction of the unit. To do so, two questionnaires based on the Net Promoter Scale (NPS) were developed, whose objective is to measure the satisfaction and loyalty of consumers or users of a company or service.

In the case of the professionals we obtained $40 \%$ of the responses for promoters, 38\% for neutral and $22 \%$ for detractors. Thus, the final NPS index for professionals was $18 \%$ and it could be classified in the improvement zone. For the patients, we obtained $62 \%$ of the responses for promoters, $35 \%$ for neutral and 3\% for detractors, and the final NPS index was 58\% and it was possible to classify it in the quality zone. 
The disparity between the two clients of the cardiology emergency unit, professionals and patients, is highlighted, which shows that patients are more likely to indicate the service to other clients when compared to professionals. We suggest a better understanding of the reasons why the results of the questionnaires applied did not reach the zone of excellence.

The situational diagnosis was made through the team's observational analysis, the results of the questionnaires applied and the study of the ombudsman cases. The weaknesses and opportunities for improvement found are mainly linked to reduced human resources, high user demand and the unit's old physical structure.

Keywords: quality management in health; patient satisfaction; health personnel; emergency care services

\section{INTRODUÇÃO}

Um serviço de pronto-atendimento é caracterizado como uma unidade intrahospitalar preparada para atender urgências e emergências (VECINA NETO, 2011), funcionando ininterruptamente para atender demandas de saúde de uma população.

Com uma média de 1138 atendimentos mensais, a unidade de emergência cardiológica (UEC) dedica-se exclusivamente ao atendimento do paciente cardiopata ou pneumopata em fase aguda da doença, preferencialmente de alta complexidade e mediante referenciamento de outras unidades de saúde (atenção primária ou secundária).

Além de demandas advindas de SAMU, Polícia Civil, Corpo de Bombeiros e casos mais graves que se apresentam à porta do serviço (demanda espontânea). O serviço é responsável pelos atendimentos advindos do Sistema Único de Saúde (SUS) e também atende pacientes da saúde suplementar, como particulares e convênios.

Na busca pela qualidade excepcional de atendimento, deve-se usar indicadores que possibilitem a avaliação da qualidade do serviço. Segundo SOUZA E SCATENA (2014), a satisfação do paciente é um indicador que pode auxiliar na avaliação da qualidade funcional da assistência hospitalar. Embora não seja o único, a satisfação do paciente tem sido usada como indicador de qualidade já que orienta a análise da assistência através da mensuração qualitativa da percepção do paciente sobre a sua estadia hospitalar. É importante considerar que o paciente sofre influência das experiências anteriores, dos cuidados médicos recebidos e do seu estado atual de saúde e que, portanto, sua percepção é fruto do balanço entre expectativa e experiência. Além disso, o serviço médico tem como característica a 
simultaneidade (PAES DE PAULA, 2005), ou seja, um serviço só é realizado na presença do profissional e do paciente. Assim, a qualidade do serviço é avaliada por completo quando analisadas ambas as partes do processo: médico e paciente.

O hospital em que a UEC está inserida possui uma pesquisa de satisfação do paciente nas unidades de internação em enfermarias e nos ambulatórios, ficando a emergência à parte da análise. Por ser um serviço de alta criticidade e variabilidade, o Pronto Socorro exige métodos de aplicação de questionários mais específicos e perguntas que levam em consideração o atual estado de saúde crítico do paciente.

Dessa forma, para avaliar e possivelmente otimizar a qualidade da assistência fornecida na unidade, faz-se necessário, primeiramente, um diagnóstico situacional do setor, através da coleta de dados e da análise de indicadores acerca da satisfação da assistência, pelo prisma dos pacientes e dos profissionais atuantes no serviço (médicos, enfermeiros e técnicos de enfermagem). A análise de percepção desses stakeholders visa o diagnóstico das possíveis causas detratoras da qualidade do serviço.

\section{OBJETIVO}

Realizar o diagnóstico situacional da UEC de forma a avaliar a qualidade da assistência fornecida ao paciente, sob a ótica da satisfação dos pacientes e também dos profissionais do serviço.

\section{MÉTODOS}

Para a realização do diagnóstico situacional da UEC, foi utilizada a satisfação percebida na unidade por pacientes e profissionais como parâmetro. Para a mensuração, foram elaborados dois questionários, um a ser aplicado nos profissionais que atuam na unidade e outro nos pacientes, sendo escolhido $o$ número de 60 para pacientes e 60 para profissionais entrevistados. Os dois questionários possuem estrutura semelhante, sendo um o espelho do outro, a fim de conseguir fazer uma análise em paralelo da percepção da satisfação do paciente sob dois pontos de vista distintos.

Para a elaboração do questionário, fundamentalmente ponderou-se a utilização da Escala NPS (Net Promoter Scale), metodologia criada por REICHHELD (2011) cujo objetivo é mensurar a satisfação e a fidelidade dos consumidores ou usuários de uma empresa ou serviço. É uma metodologia caracterizada por sua simplicidade e confiabilidade por se tratar de uma pergunta simples: "Em uma escala de 0 a 10, o quanto você recomendaria a empresa $X$ para um amigo ou colega?", onde as respostas variam numa escala de 0 a 10 , sendo 0 a menor recomendação e 10 a maior recomendação.

Para o cálculo das respostas com base nas notas de 0 a 10, dividem-se os clientes em três categorias: 
- Promotores: pontuam com notas 9 e 10. São clientes que passaram a ter benefícios após seu relacionamento com a marca ou empresa, oferecem feedback positivo, são leais e voluntariamente divulgam a empresa;

- Neutros: pontuam com notas 7 e 8, são aqueles que se utilizam da empresa apenas por necessidade, sem lealdade ou entusiasmo com a marca;

- Detratores; pontuam com notas de 0 a 6 , são os clientes que não recomendam a empresa, criticam em público e não voltariam a fazer negócio, sendo influenciadores negativos da marca.

A partir desta categorização, são somadas as notas e calculada a porcentagem de cada uma das categorias diante do geral. O NPS é calculado através da subtração entre a porcentagem de clientes promotores e detratores. $O$ valor obtido pode ser classificado em quatro zonas de avaliação da empresa ou marca:

- Entre 75 e 100: zona de excelência;

- Entre 50 e 75: zona de qualidade;

- Entre 0 e 50: zona de aperfeiçoamento;

- Entre -100 e 0: zona crítica.

A pedido da UEC, a Escala NPS foi adaptada, passando de pontuações de 0 a 10 a quatro categorias simbolizadas por faces que expressavam "ótimo" (equivalente às notas 9 e 10), "bom" (equivalente às notas 7 e 8), "médio" (equivalente às notas 5 e 6) e "ruim" (equivalente às notas de 0 a 4).

Além da pergunta essencial, o questionário continha outras quatro perguntas, relativas ao retorno do paciente ao serviço em caso de posterior necessidade, sobre a assistência do serviço, sobre a assistência recebida pelo paciente e sobre sugestões de melhoria, pergunta aberta para que os entrevistados pudessem apontar os pontos a melhorar no serviço.

Para os pacientes entrevistados, foram utilizados os seguintes critérios de inclusão:

- Ter mais de 20 anos;

- Estar estável hemodinamicamente e consciente;

- Internado há pelo menos 24 horas na UEC e no máximo 48 horas;

- Vínculo SUS ou convênio/particular.

Após a coleta, foi feita a análise dos resultados e formulação do diagnóstico situacional da UEC e da qualidade assistencial de seu serviço sob a percepção 
dos pacientes e dos profissionais, pontuando, caso houvesse, as possíveis causas detratoras.

\section{RESULTADOS}

\section{Entrevistas com profissionais e pacientes}

Em relação à formação profissional dos entrevistados, $45 \%$ foram auxiliares/técnicos de enfermagem, $40 \%$ médicos, $8 \%$ SOS/recepção e $7 \%$ enfermagem, totalizando 60 entrevistados.

Quanto aos pacientes, os maiores estratos dos 60 entrevistados foram do sexo masculino (63\%), entre 56 e 65 anos de idade (32\%), e com escolaridade de ensino fundamental incompleto (42\%).

Em relação ao item "origem", ou seja, local prévio do paciente à UEC, 62\% deles eram provenientes da própria residência, índice esse que contradiz a postura referenciada da UEC.

Para o item "vínculo", 92\% dos pacientes eram do SUS e apenas $8 \%$ eram usuários da saúde suplementar. Segundo o relatório de março de 2017 da unidade de informações médicas e hospitalares (UIMH) do hospital, 34,8\% das consultas realizadas na UEC (total de 1191) foram de convênio. Essa disparidade numérica encontrada entre o relatório mensal da UIMH e os resultados do atual estudo pode ser explicada pela baixa taxa de internação dos pacientes da saúde suplementar quando comparadas às do SUS, visto que para a saúde suplementar a porta é aberta e no SUS há o referenciamento. Como o tempo de estadia mínimo de 24 horas era premissa para a entrevista, a vasta maioria dos pacientes enquadrados nas condições necessárias deste estudo foi proveniente do SUS.

Quando perguntados se retornariam ao serviço no caso de emergências e de livre escolha entre todos os serviços existentes, $87 \%$ dos profissionais responderam "SIM", e apenas $13 \%$ responderam "NÃO". Quanto aos pacientes, as respostas foram respectivamente $95 \%$ e $5 \%$, conforme ilustrado abaixo (Gráfico 1). 


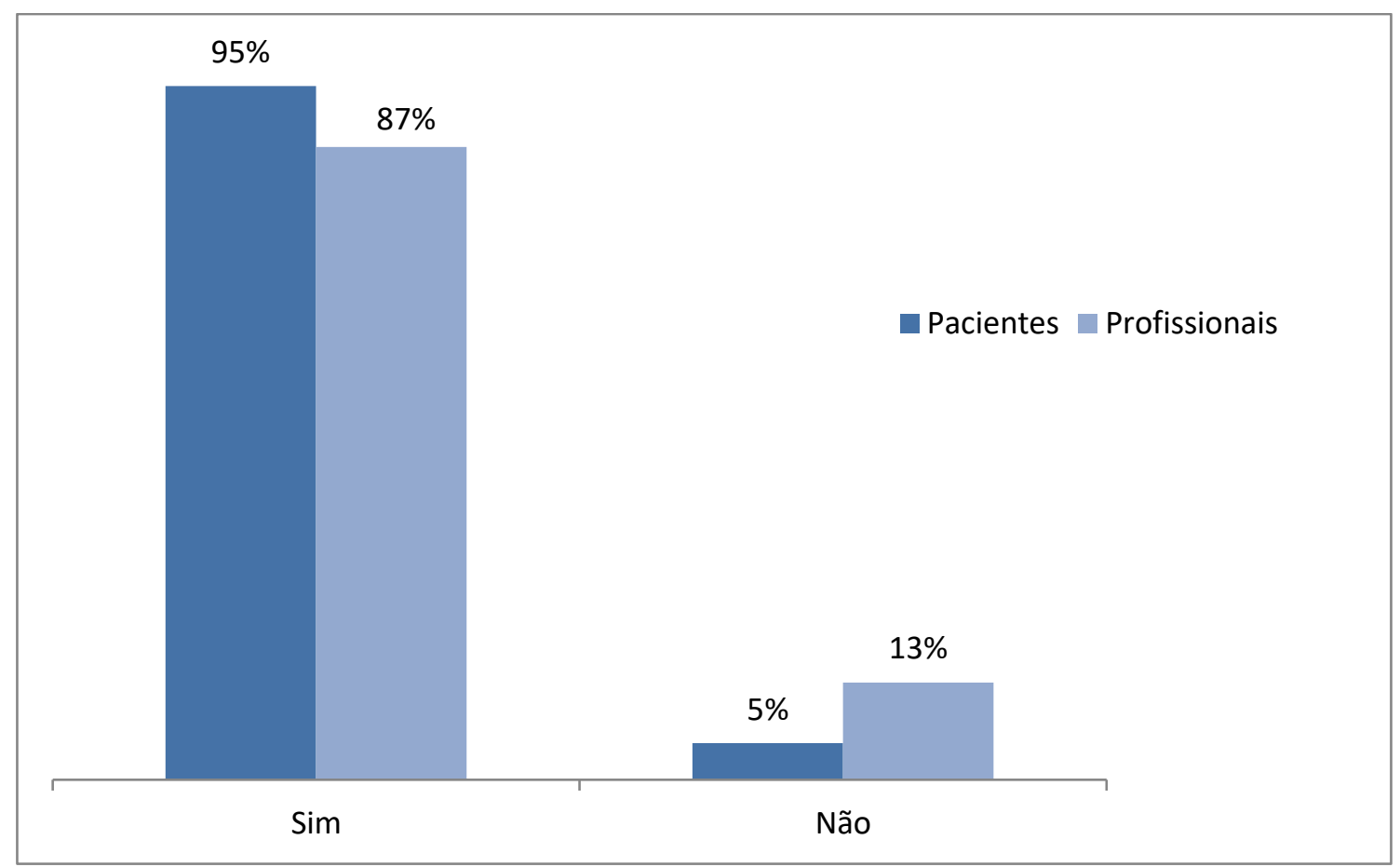

Gráfico 1. Distribuição de respostas de pacientes e profissionais quando perguntados se retornariam ao serviço no caso de livre escolha entre instituições de saúde da cidade. Total de 60 pacientes e 60 profissionais.

Quando perguntados sobre recomendar o serviço a seus familiares e amigos, na categoria dos profissionais, obteve-se $40 \%$ das respostas para a opção "ótimo" (promotores), 38\% "bom" (neutros), 22\% "médio" (detratores) e 0 para "ruim" (detratores). Seguindo a análise do NPS explicada anteriormente, o índice final do NPS para os profissionais foi de $18 \%$, sendo possível classificalo na zona de aperfeiçoamento. Para os pacientes da UEC, as respostas somaram, respectivamente $62 \%$ das respostas para a opção "ótimo" (promotores), 35\% "bom" (neutros), 3\% "médio" (detratores) e 0 para "ruim" (detratores), tendo um índice NPS de 58\%, sendo classificado na zona de qualidade.

Ressalta-se nesse ponto a disparidade entre os dois clientes da UEC: profissionais e pacientes. Enquanto os primeiros apresentaram um NPS de $18 \%$, o segundo grupo apresentou $58 \%$, o que demonstra que os pacientes são mais propensos a indicarem o serviço para outros clientes quando comparados aos profissionais. Isso é favorecido também pela questão da percepção da qualidade assistencial prestada dos profissionais. Por acreditarem que não estão prestando um bom serviço, tendem a indicar menos o serviço.

Em relação à pergunta sobre qualidade da assistência oferecida pelo UEC aos seus pacientes, 90\% respondeu que "SIM", $8 \%$ que "NÃO" e $2 \%$ não respondeu a essa pergunta. Para os pacientes, as respostas foram, respectivamente $97 \%$ e 3\%, conforme o Gráfico 2 mostrado abaixo. 


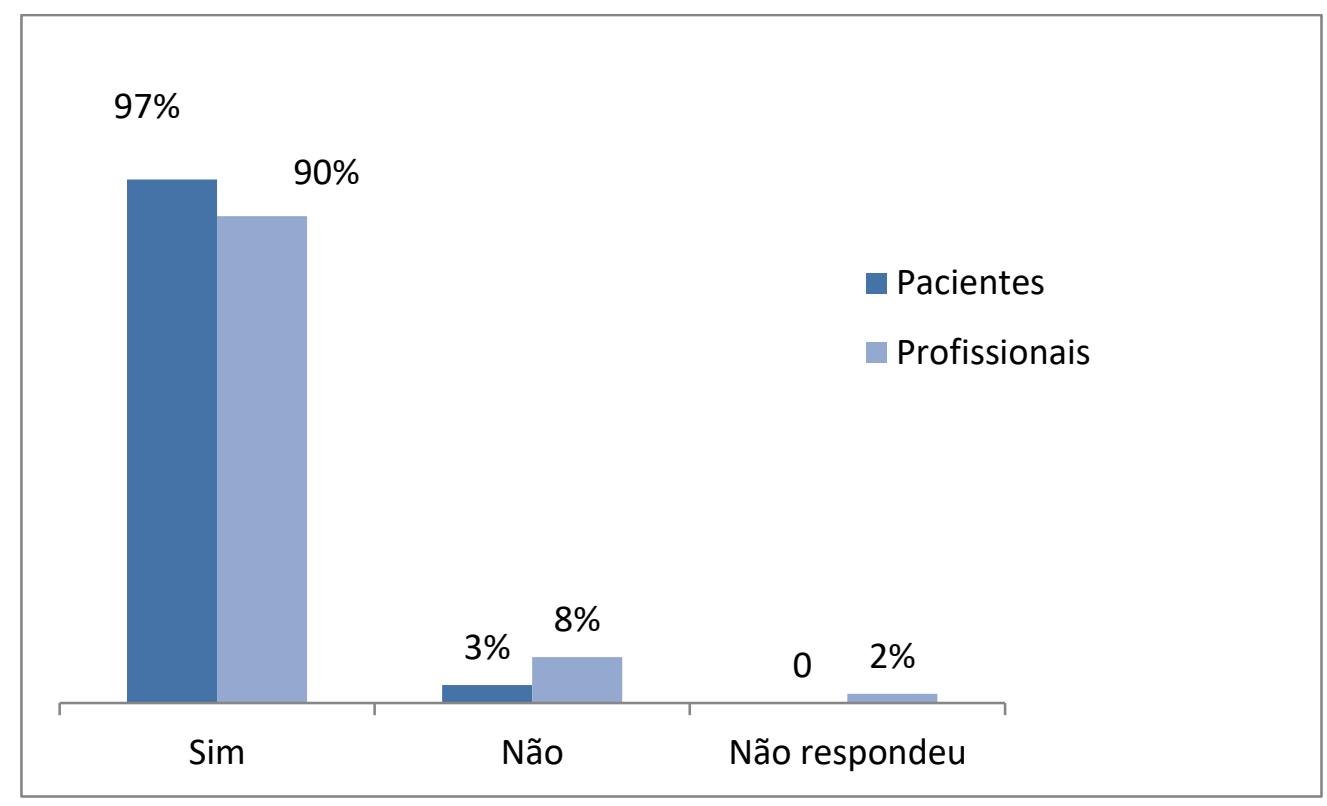

Gráfico 2. Distribuição de respostas de pacientes e profissionais quando perguntados sobre se há qualidade na assistência prestada pelo serviço. Total de 60 pacientes e 60 profissionais.

Na pergunta sobre a prestação de uma assistência de qualidade dos profissionais e sua pergunta espelhada aos pacientes sobre a assistência recebida durante aquela internação, houve um equilíbrio entre as respostas, sendo que $57 \%$ dos profissionais responderam "SIM", ou seja, que conseguem realizar uma assistência de qualidade a todos os pacientes. No entanto, $43 \%$ deles responderam "NÃO". Para os pacientes, a assistência recebida foi $93 \%$ de respostas positivas e $7 \%$ de respostas negativas.

Essa relação entre as duas percepções pode ter algumas explicações como o grau de autoexigência dos profissionais, disparidades nas expectativas criadas pelos dois grupos, influência do grau de escolaridade, desconhecimento por parte dos pacientes de outros serviços, entre outros.

Ao se estratificar a resposta dos profissionais, pode-se concluir que a categoria profissional mais insatisfeita com seu desempenho, em termos absolutos, foi a dos auxiliares/técnicos de enfermagem (15 profissionais dentro dos 27, correspondendo a 56\%). Em termos relativos, a classe mais afetada foi a da enfermagem (3 profissionais dentro do total de 4 , correspondendo a $75 \%$ ).

Tabela 1. Estratificação dos profissionais insatisfeitos com o seu desempenho profissional.

\begin{tabular}{|l|l|l|}
\hline $\begin{array}{l}\text { Respostas } \\
\text { "NÃO" }\end{array}$ & $\begin{array}{l}\text { Total entrevistado } \\
\text { por categoria } \\
\text { profissional }\end{array}$ & $\%$ \\
\hline
\end{tabular}




\begin{tabular}{|l|r|r|r|}
\hline Auxiliar/técnico & 15 & 27 & $56 \%$ \\
\hline Enfermeiro & 3 & 4 & $75 \%$ \\
\hline Médico & 7 & 24 & $29 \%$ \\
\hline
\end{tabular}

Foram obtidas 38 respostas de um total de 60 para a questão referente à sugestão de melhoria, sendo que cada uma das respostas poderia conter mais de uma sugestão. Após análise das respostas obtidas, cada uma das sugestões foi categorizada em uma das seguintes classes: rede de saúde; integração das equipes (médico $X$ equipe de enfermagem e auxiliares/técnicos de enfermagem); informatização da assistência; gerenciamento; humanização; educação continuada; valorização do $\mathrm{RH}$; tempo; estrutura para paciente; acesso; remuneração; estrutura física; aumento de $\mathrm{RH}$.

A sugestão mais frequente foi a de aumento de $\mathrm{RH}(29 \%)$, seguida pela de melhora da estrutura física do PS (17\%) e pela de aumento da remuneração dos profissionais (12\%).

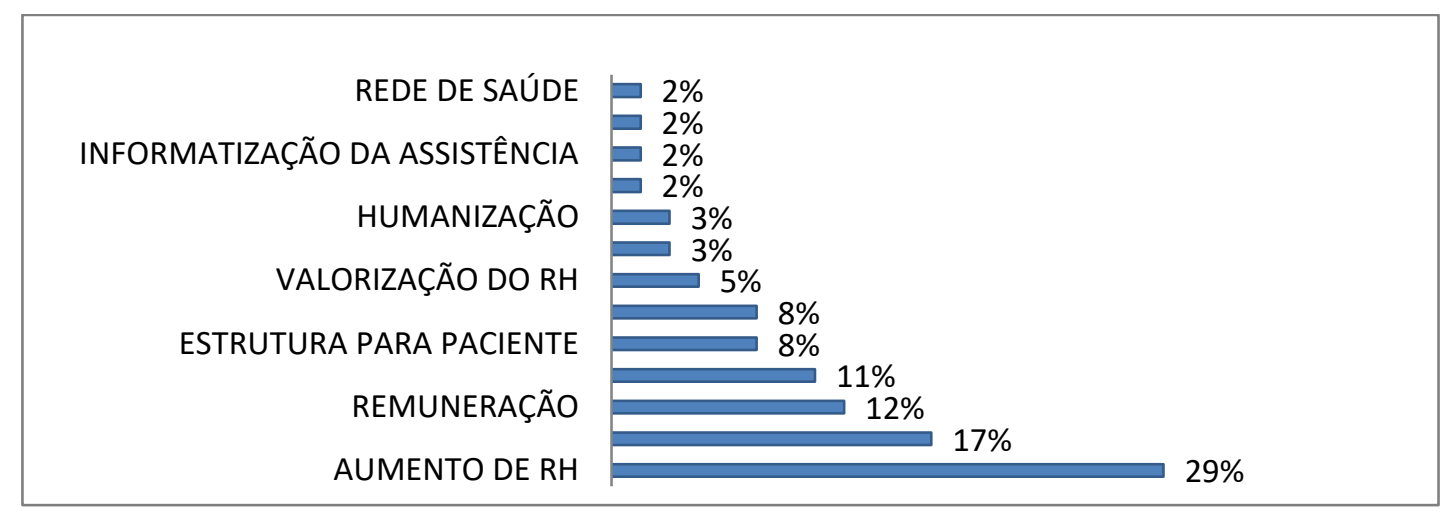

Gráfico 3. Sugestões de melhoria para a unidade de emergência de acordo com os profissionais.

Para fins de análise da relação de causa/efeito entre as sugestões de melhoria (causa) e os números obtidos sobre qualidade da assistência prestada aos pacientes (efeito), foi realizada uma análise estatística com o teste quiquadrado para todas as variáveis relacionadas.

Tabela 2. Valores de significância estatística entre as sugestões de melhoria e a percepção de qualidade de assistência prestada pela unidade.

\begin{tabular}{|l|r|}
\hline Sugestão de melhoria & p-value \\
\hline Acesso & $\mathbf{0 , 0 2}$ \\
\hline Estrutura física & 0,11 \\
\hline
\end{tabular}




\begin{tabular}{|l|r|}
\hline Tempo & 0,17 \\
\hline Estrutura para paciente & 0,17 \\
\hline Humanização & 0,33 \\
\hline Valorização do RH & 0,49 \\
\hline Gerenciamento & 0,50 \\
\hline Remuneração & 0,93 \\
\hline Rede de saúde & 0,94 \\
\hline Integração das equipes & 0,94 \\
\hline Informatização da assistência & 0,94 \\
\hline Educação continuada & 0,94 \\
\hline Aumento de RH & 1,00 \\
\hline
\end{tabular}

Dessa forma, pode-se observar que apenas a variável "acesso" foi estatisticamente significante ( $p$-value 0,02). Quando analisadas as respostas categorizadas em "acesso" é possível notar duas vertentes principais, ambas relacionadas ao acesso de atendimento médico. A primeira se correlaciona ao atendimento do paciente e o referenciamento da unidade, ou seja, os profissionais acreditam que para poderem prestar uma assistência de qualidade a todos os pacientes da UEC, o modelo de serviço referenciado para casos cardiológicos graves com respeito à hierarquização preconizada pelo SUS deve funcionar rigorosamente. Pacientes que procuram a UEC que não se enquadram nas perspectivas de complexidade exigidas acabam por prejudicar o serviço como um todo.

A segunda vertente refere-se ao atendimento médico da própria equipe de profissionais que trabalham na UEC, haja vista a oferta inexistente de cuidados à saúde do profissional nas dependências da UEC.

Por mais que não tenham sido estatisticamente significantes para a relação de causa/efeito com o índice baixo de "SIM" $(56,67 \%)$ para a pergunta de qualidade da assistência prestada aos pacientes da UEC as demais sugestões propõem dados interessantes no que tange a pontos fracos e ameaças ao serviço, que devem ser levadas em consideração para planos de ações futuros em prol de se atingir a excelência do serviço.

No caso dos pacientes, apenas 22 do total de 60 entrevistados responderam a questão de número 5 , referente a sugestões de melhorias para o serviço da UEC. Assim como no questionário dos profissionais, as respostas dos pacientes também foram categorizadas em algumas classes: tempo; ambiência; comunicação; acesso; estrutura para paciente. 


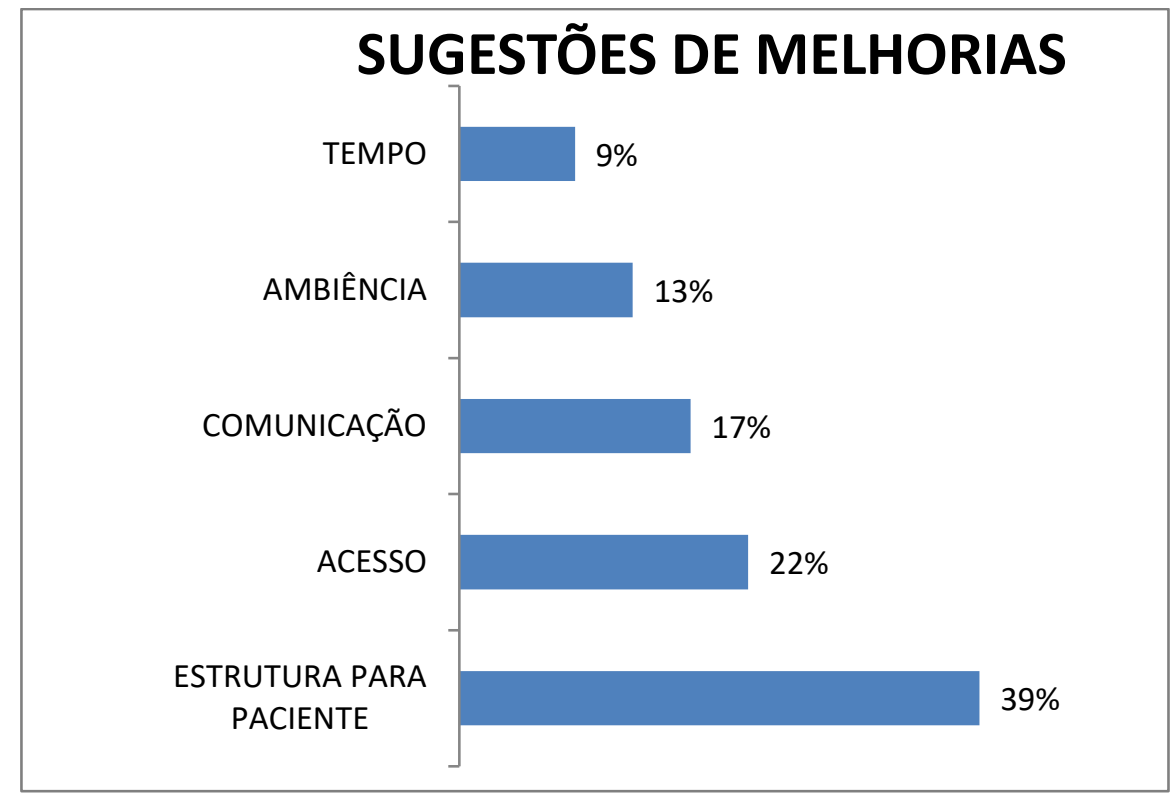

Gráfico 4. Sugestões de melhorias para a unidade de emergência segundo os pacientes entrevistados.

A sugestão mais prevalente foi a de melhorar a estrutura física para o paciente internado e seu acompanhante (39\%). Questões como dificuldade de acessibilidade ao banheiro, tamanho do pronto socorro, hotelaria deficiente (inexistência de itens como televisores e aparelhos de ar condicionado), baixo número de leitos para o volume de pacientes e ausência de cadeiras para os acompanhantes foram evidenciadas pelos questionários. Diferentemente dos profissionais, aqui o item "acesso", correspondendo a $22 \%$ das sugestões, diz respeito à dificuldade de acesso ao atendimento na UEC. Para os pacientes, 0 referenciamento prejudica e dificulta a assistência médica do SUS. Em terceiro lugar, o item "comunicação" (17\%) refere-se ao baixo volume de informação oferecido pelos profissionais aos pacientes em relação ao seu diagnóstico, conduta e prognóstico. No quesito "ambiência" (13\%), as queixas foram sobre o barulho do ambiente, a confusão e o grande volume de pacientes internados.

\section{Ouvidoria do paciente}

Foram obtidos 61 casos de ouvidoria dentro do período de 05/01/2016 e 29/12/2016. Mais da metade dos casos foram enviados via site ou pessoalmente, conforme mostra Tabela 3.

Tabela 3. Distribuição das formas de comunicação do paciente com a ouvidoria do hospital.

\begin{tabular}{|l|r|}
\hline Forma & Quantidade \\
\hline Carta & 5 \\
\hline E-mail & 7 \\
\hline
\end{tabular}




\begin{tabular}{|l|r|} 
Formulário de pesquisa & 3 \\
\hline Pessoalmente & 21 \\
\hline Site & 25 \\
\hline Total Geral & 61 \\
\hline
\end{tabular}

Os casos da ouvidoria foram obtidos com o intuito de se realizar um paralelo entre as sugestões de melhorias dos pacientes internados na UEC com as queixas/elogios dos pacientes que já experimentaram o serviço e não estão mais internados. Da mesma forma que nos questionários aplicados, os dados da ouvidoria também foram categorizados em classes.

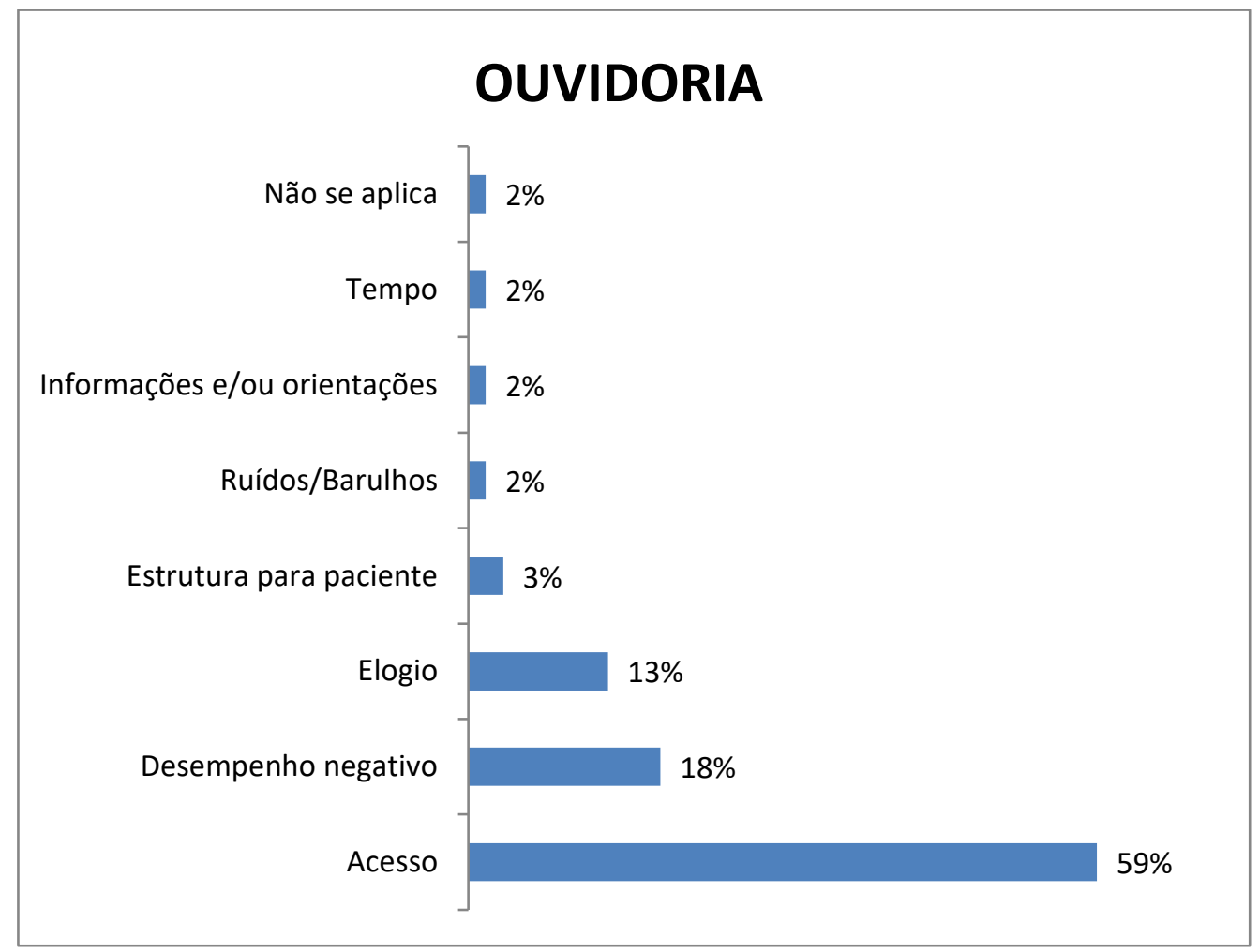

Gráfico 5. Distribuição dos motivos de comunicações de pacientes recebidas pela ouvidoria do hospital.

Enquanto que a dificuldade de acesso ao serviço devido ao referenciamento foi de $22 \%$ para os pacientes internados, $59 \%$ dos casos da ouvidoria contemplaram tal classe, ficando como queixa mais prevalente. Em segundo lugar, $18 \%$ acredita ter sido exposto a situações em que o desempenho do serviço foi abaixo do esperado, seja por incapacidade técnica, seja por abuso moral. Houveram 8 elogios dentro dos 61 casos da ouvidoria. Além destes, casos como falta de estrutura para o paciente $(3 \%)$, ruídos e barulhos $(2 \%)$ e 
tempo de espera excessivo (2\%) também estavam presentes nos dados da ouvidoria.

Os casos referentes ao item "não se aplica" foram classificados de tal forma, pois não eram referentes à UEC. Já a classe "informações e/ou orientações" contempla os casos de ouvidoria que eram apenas dúvidas sobre o funcionamento do serviço.

\section{Diagnóstico situacional}

Após a pesquisa foi feito o diagnóstico situacional da UEC, cuja função foi a de estabelecer uma análise detalhada da situação atual e auxiliar no planejamento estratégico e na tomada de decisões em prol da melhoria do serviço.

Vale destacar como pontos fortes a alta qualificação dos profissionais da UEC, a imagem nacional e internacionalmente conhecida de centro especializado de excelência e referência em cardiologia, o referenciamento do serviço e a avaliação positiva pelos pacientes e profissionais em relação à qualidade da assistência, como pôde ser visto na análise dos questionários aplicados.

Para se atingir a excelência no atendimento, deve-se aproveitar a oportunidade da mudança do espaço físico da UEC. $17 \%$ dos profissionais apontaram a estrutura física da UEC como um ponto de melhoria, 39\% dos pacientes pontuaram a melhora da estrutura física como sugestão e 3\% dos dados da ouvidoria elencavam tal ponto. Junto com a mudança, os fluxos de atendimento e procedimentos devem ser otimizados e novos equipamentos médicoassistenciais adquiridos de forma a absorver a nova demanda de pacientes, e ofertar uma experiência que supra as novas expectativas. Em relação ao referenciamento, fator importante como causa da percepção da assistência prestada de baixa qualidade pelos profissionais, deve-se aproveitar a divulgação do novo PS para classificá-lo como serviço referenciado especializado, de forma a evidenciar os benefícios de tal decisão a todos os usuários e reforçar a sua importância.

Os pontos fracos e as ameaças foram aqui pontuados através da análise observacional da equipe, dos resultados dos questionários aplicados e do estudo dos casos de ouvidoria. São de suma importância a compreensão e a definição desses pontos para a formulação de planos de ações eficientes que abordem todos os fatores detratores do serviço. Foram assim definidos:

1. Recursos humanos reduzidos;

2. Grande demanda de usuários;

3. Estrutura física da UEC (antiga);

4. Ambiência;

5. Comunicação deficitária entre a equipe e os pacientes; 
6. Sensação de desvalorização do $\mathrm{RH}$;

7. Não compreensão do modelo de referenciamento pela população;

8. Desempenho negativo de alguns profissionais segundo dados da ouvidoria;

9. Nova estrutura física e ruídos da mudança;

10. Divulgação na mídia de maneira desfavorável;

11. Divulgação errônea do referenciamento;

12. Equipe insatisfeita com a qualidade da assistência prestada;

13. Remuneração insuficiente;

14. Concorrência com outros serviços em relação a saúde suplementar.

Os recursos humanos reduzidos foram evidenciados como queixa dos profissionais, e como consequência desse cenário há diminuição do desempenho por aumento de carga laboral e indefinição das funções específicas de cada profissional que pode acarretar em baixo desempenho pessoal.

\section{CONSIDERAÇÕES FINAIS}

Por mais que o serviço seja referenciado, ainda há um grande número de pacientes que são atendidos na UEC. Segundo os dados da UIMH, a média mensal de atendimentos na UEC é de 1138. Para acatar tal demanda, deve haver um espaço físico que comporte todos os usuários, um número de profissionais suficiente para o atendimento de qualidade e fluxos bem definidos.

A mudança física da UEC foi para o serviço do SUS. A saúde suplementar do hospital continua sendo ofertada na estrutura antiga, que apresenta um importante déficit de hotelaria e uma ambiência desalinhada com os padrões esperados em um hospital.

A comunicação deficitária entre a equipe e os pacientes é um fator que deve ser levado em consideração já que é um direito do paciente e um dever da equipe médica manter o usuário constantemente atualizado sobre seu prognóstico e conduta.

A desvalorização do $\mathrm{RH}$ reflete a falta de reconhecimento financeira e laboral dos profissionais que trabalham no serviço. Segundo os questionários aplicados, há uma ausência de feedback positivo e de remuneração justa. 
A não compreensão da população sobre o referenciamento leva a insatisfação do usuário que não consegue receber atendimento e ao aumento da demanda interna de atendimentos de pacientes que não deveriam ser atendidos por esse serviço, o que acaba prejudicando a qualidade da assistência prestada.

Por mais que a nova estrutura física do serviço deva sanar alguns problemas de ordem estrutural como falta de banheiros, espaços reduzidos e falta de cadeiras para acompanhantes, um novo ambiente, em que os fluxos não estão completamente estabelecidos sempre leva a desorganização e diminuição de eficiência. Deve-se então se atentar para os possíveis ruídos gerados na nova unidade de emergência.

A divulgação do referenciamento e da nova UEC devem estar associados. Como já foi dito anteriormente, é fundamental pontuar a todos os benefícios de um serviço referenciado e dessa forma otimizar o atendimento dos pacientes cardiológicos graves.

Entender o funcionamento do referenciamento e a sua importância para um atendimento de qualidade com uma demanda específica foi de grande valia para a equipe de projeto, possibilitando uma visão integral acerca da Unidade.

Acerca da pesquisa de satisfação e qualidade da assistência prestada para o paciente, entende-se que este é um indicador muito importante para a manutenção de um serviço de excelência e que atenda às necessidades do paciente em sua completude. Trabalhos como este contribuem para a melhoria do processo de gestão da UEC e da qualidade da assistência e busca constante pela excelência.

\section{REFERÊNCIAS}

Castanheira, CHC, Pimenta AM, Lana FCF, Malta, DC. Utilização de serviços públicos e privados de saúde pela população de Belo Horizonte. Rev. Bras. Epidemiol. [Internet]. 2014; 17 (Suppl 1): 256-266. Disponível em: http://www.scielo.br/scielo.php?script=sci arttext\&pid=S1415790X2014000500256\&lng=en.

Paes de Paula, A. P. (2005). Administração Pública Brasileira entre o Gerencialismo e a Gestão Social. Revista de Administração de Empresas, 45 (1), 37-49.

Souza, PC, Scatena, JHG. Satisfação do usuário da assistência hospitalar no mix público-privado do SUS do Estado de Mato Grosso. Revista Espaço Para a Saúde. Londrina, v. 15, n. 3, p. 30-41, jul./set. 2014. Disponível em: http://www.uel.br/revistas/uel/index.php/espacoparasaude/article/viewFile/1804 $\underline{8 / p d f ~} 38$

Reichheld, Fred; Markey, Rob. The Ultimate Question 2.0 (Revised and Expanded Edition): How Net Promoter Companies Thrive in a Customer-Driven World. Harvard Business Review Press; 2011; 304p. 
Vecina Neto, G, Malik, AM. Gestão em Saúde. Rio de Janeiro: Guanabara Koogan; 2011. 400p.

Recebido: 16 de maio de 2018. Publicado: 26 de outubro de 2018

Correspondência: Ana Cláudia Becker. E-mail: becker.anac@gmail.com

Conflito de Interesses: os autores declararam não haver conflito de interesses.

(C) This is an Open Access article distributed under the terms of the Creative Commons Attribution License, which permits unrestricted use, distribution, and reproduction in any medium, provided the original work is properly cited 